\title{
Childhood blindness due to vitamin A deficiency in India: regional variations
}

\author{
J S Rahi, S Sripathi, C E Gilbert, A Foster
}

\begin{abstract}
Objectives-To investigate the importance of vitamin $A$ deficiency as a cause of childhood blindness and severe visual impairment (SVI) in India.

Design and setting-Cross sectional survey of children with visual acuity less than 6/60 in the better eye (SVI/blindness), attending 22 schools for the blind in nine states of India.

Main outcome measure-Proportion of children with blindness or SVI from corneal scarring attributable to vitamin $A$ deficiency.

Results-245 of $1318(18 \cdot 6 \%)$ of children had SVI/blindness attributable to vitamin A deficiency. The proportion of SVI/blindness due to vitamin $A$ deficiency ranged from $7 \cdot 5 \%(7 / 93)$ in Kerala to $26 \cdot 7 \%$ (27/101) in Madhya Pradesh. In Tamil Nadu, $7 \cdot 5 \%(9 / 120)$ of children in the capital city blind school had SVI/blindness due to vitamin A deficiency, compared with $30.4 \%(51 / 168)$ in a blind school in a rural area of the same state.

Conclusion-Overall, vitamin A deficiency is the single most important cause of childhood blindness and SVI in India. There are marked variations by state and also between urban and rural locations. (Arch Dis Child 1995; 72: 330-333)
\end{abstract}

Keywords: blindness, vitamin A deficiency, India.

An estimated 0.5 million children become blind in the world each year and $60-80 \%$ die within two years of the onset of blindness (report of World Health Organisation (WHO) meeting, 1990). The most common cause of visual loss in children in developing countries is vitamin A deficiency, which accounts for up to $70 \%$ of cases in some countries. Data on childhood blindness due to vitamin A deficiency in India are incomplete and standardised data comparing patterns of blindness in different states are scarce. India is an industrialising nation with variation by state in socioeconomic factors that are relevant to the occurrence of vitamin A deficiency.

The contribution of vitamin A deficiency as a factor in childhood mortality is now widely accepted. ${ }^{1}$ The ocular complications of vitamin A deficiency are well documented and often used as clinical indicators of current and past vitamin A status.

The magnitude of visual loss resulting from vitamin A deficiency is less easily estimated. It is, however, probably the most important worldwide cause of blindness in children. The
WHO estimates that 13.8 million children worldwide have some degree of visual loss attributable to vitamin A deficiency. ${ }^{2}$

A few cross sectional surveys have estimated the prevalence of blindness associated with vitamin A deficiency to be anything from $0.4 \%$ to $3.4 \%$ in preschool children in Asia. ${ }^{3}$ In India approximately 15000 preschool children are blinded each year by lack of vitamin $A .{ }^{4}$

Vitamin A deficiency is usually seen in combination with protein energy malnutrition and acute systemic illnesses notably diarrhoeal diseases and measles. ${ }^{5}$ The interaction of biological, nutritional, and socioeconomic factors resulting in vitamin A deficiency is complex and this is reflected in its varying prevalence in different communities.

India has a system of federal government and this is reflected in public health planning and implementation. The rapid industrialisation of parts of the country has contributed to the wide range of socioeconomic conditions seen.

A review of the prevalence of clinical vitamin A deficiency in preschool children estimated a range from $4 \cdot 4 \%$ to $34 \cdot 1 \%$ in different regions of the country. ${ }^{6}$

Data on blindness due to lack of vitamin A have uses in the planning of ophthalmic services at central and state level as well as in formulation of public health policies to combat the deficiency and its associated morbidity and mortality.

\section{Methods}

The aim of the study was to determine the major causes of severe visual impairment and blindness in Indian children attending schools for the blind. The standard WHO classification of visual loss was employed. ${ }^{7}$ Severe visual impairment (SVI) refers to a corrected visual acuity, in the better eye, of less than $6 / 60$ but equal to or better than $3 / 60$, while blindness refers to a corrected visual acuity, in the better eye, of less than $3 / 60$.

It is estimated that 15000 children attend residential schools for the blind in India and a further 5000 are in integrated education (P G Michael, personal communication, 1993). These children, therefore, represent approximately $10 \%$ of the estimated 200000 blind children in India. ${ }^{8}$

Schools for the blind were selected nonrandomly in order to meet the objective of examining a minimum of 100 children in each state between the ages of 3-15 years, of both sexes, and representing both urban and rural backgrounds. All schools were initially identified from the Resource Directory of the International Council for the Education of the 
Visually Handicapped ${ }^{9}$ and by discussion with liaising ophthalmologists in India. In some states, no schools could be identified. Time and physical constraints on travel also imposed limitations on the study size and coverage. Twenty two schools were eventually selected in nine states in different geographical zones and a study size of approximately $10 \%$ of the estimated blind school population of India was achieved.

Each school principal was sent a written request for permission to visit the school and carry out ophthalmic examinations of all children attending it. An outline of the purpose of the study accompanied the written request to visit. Concurrently, ophthalmologists associated with the nearest large ophthalmic unit were contacted to request support, advice, and assistance with the study. All schools contacted agreed to inclusion of their pupils in the study. Where feasible, parents were present on the day their child was examined.

The 22 schools in nine states were visited between mid-March and mid-August 1993.

Each child was seen with the class teacher and parent, when available. Sociodemographic data - age, sex, place of residence, area of birth, ethnic group, and caste or paternal occupation - were recorded for each child. A routine medical history was taken including age at onset of visual loss, previous or current medical or surgical interventions, other disabilities or relevant clinical features. Any relevant details of family or obstetric history were noted. Where information was lacking, for example in the case of orphaned children, medical records held for each child with school registration documents were examined. Such records were usually available as an ophthalmologist's report is a prerequisite for admission to most schools.

Each child was examined by the study optometrist who measured visual acuity using an illiterate $\mathrm{E}$ chart, the visual fields (by confrontation), and function vision such as the ability to recognise faces, to walk unaided, or to discern large print. The children were routinely refracted after cycloplegia.

Each child was then seen by the study ophthalmologist. Anterior segment examination was carried out with a magnifying loupe and torch. Posterior segment examination was carried out by direct and/or indirect ophthalmoscopy after pupillary dilation.

The findings were recorded on the WHO prevention of blindness eye examination record for children with blindness and low vision. ${ }^{10}$ This form allows categorisation of cause of visual loss by anatomical site and by aetiology.

A clinical diagnosis of xerophthalmia was made in children with typical corneal scars (leucoma, adherent leucoma, staphyloma) or phthisis bulbi in whom the medical history, including age of onset and associated diseases, strongly supported the diagnosis. For each child any necessary optical, surgical, or medical interventions were recorded.

A report of the findings and recommendations at each school was given to the school
Table 1 Proportion of children with SVI/blindness due to vitamin $A$ deficiency by state in 1318 children in nine states of India, 1993

\begin{tabular}{|c|c|c|c|}
\hline \multirow[b]{2}{*}{ State } & \multirow{2}{*}{$\begin{array}{l}\text { Total No } \\
\text { with SVII } \\
\text { blindness }\end{array}$} & \multicolumn{2}{|c|}{$\begin{array}{l}\text { SVI/blindness due to } \\
\text { vitamin A deficiency }\end{array}$} \\
\hline & & No (\%) & $95 \% C I$ \\
\hline $\begin{array}{l}\text { Madhya Pradesh } \\
\text { West Bengal } \\
\text { Gujarat } \\
\text { Uttar Pradesh } \\
\text { Maharashtra } \\
\text { Tamil Nadu } \\
\text { Haryana } \\
\text { Karnataka } \\
\text { Kerala }\end{array}$ & $\begin{array}{r}101 \\
89 \\
97 \\
134 \\
157 \\
384 \\
141 \\
122 \\
93\end{array}$ & $\begin{array}{l}27(26 \cdot 7) \\
22(24 \cdot 7) \\
21(21 \cdot 6) \\
29(21 \cdot 5) \\
32(20 \cdot 4) \\
71(18 \cdot 5) \\
22(15 \cdot 6) \\
14(11 \cdot 5) \\
7(7 \cdot 5)\end{array}$ & $\begin{array}{r}18.4 \text { to } 35 \cdot 6 \\
16.0 \text { to } 33.9 \\
13.4 \text { to } 30.6 \\
15.0 \text { to } 28.9 \\
13.7 \text { to } 26.3 \\
15.1 \text { to } 22.9 \\
10.0 \text { to } 22 \cdot 0 \\
6.3 \text { to } 17 \cdot 7 \\
2.5 \text { to } 13.5\end{array}$ \\
\hline Total & 1318 & $245(18 \cdot 6)$ & $16 \cdot 8$ to $21 \cdot 1$ \\
\hline
\end{tabular}

$\mathrm{CI}=$ confidence interval; $\mathrm{SVI}=$ severe visual impairment

principal and to the local liaising ophthalmologist who agreed to provide further assessment and treatment as required.

\section{Results}

A total of 1411 children were examined. The results presented relate to the 1318 children who had SVI or blindness using the WHO classification. ${ }^{7}$ Altogether $44 \%$ (573) of these children were under 11 years and 56\% (769) were between 11 and 15 years; $58 \%$ were male.

The study identified that $19 \%(245 / 1318)$ of the SVI/blindness was attributable to vitamin A deficiency. In these children the age and sex distributions were similar to the study group overall with $40 \%(96 / 245)$ of children under 11 years and $52 \%(127 / 245)$ being male.

While the proportion of children with SVI/blindness attributable to vitamin A deficiency was found to be $19 \%$ for the whole study group, the proportion varied by state. The findings by state are presented in table 1 . The range for the nine states varied from $8 \%$ in Kerala to $27 \%$ in Madhya Pradesh.

In Tamil Nadu children were examined separately in the state capital of Madras, the smaller town Madurai, and in the rural setting of Palayamkottai. Vitamin A deficiency was responsible for $8 \%$ childhood SVI/blindness in the blind school of the state capital, for $12 \%$ in the town of Madurai, and for $30 \%$ in the rural area of Palayamkottai (table 2).

\section{Discussion}

The main purpose of the study was to identify the major causes of SVI and blindness in children in India. There are methodological problems inherent in the study of children attending schools for the blind. Firstly, it is not possible to estimate prevalence rates for the population. Secondly, by exclusion of children who are not normally admitted to schools for the blind, for example those with multiple disabilities, biased estimates of the relative importance of different causes of visual impairment may result. Exclusion of preschool children means that those children who die as a result of the disease causing blindness before reaching school age are not included. While most schools in the study had pupils from urban and rural backgrounds, access is usually 
Table 2 Proportion of SVI/blindness due to vitamin A deficiency in 384 children in Tamil Nadu, 1993

\begin{tabular}{llcr}
\hline & $\begin{array}{l}\text { Total No } \\
\text { with SVI/ } \\
\text { blindness }\end{array}$ & \multicolumn{2}{l}{$\begin{array}{l}\text { SVI/blindness due to } \\
\text { vitamin A deficiency }\end{array}$} \\
\cline { 3 - 4 } \cline { 3 - 4 } Area $(\%)$ & \multicolumn{1}{c}{$95 \%$ CI } \\
\hline Madras & 120 & $9(7.5)$ & $3 \cdot 1$ to 12.8 \\
Madurai & 96 & $11(11.5)$ & $5 \cdot 5$ to 18.5 \\
Palayamkottai & 168 & $51(30.4)$ & 23.0 to 36.9 \\
Total & 384 & $71(18.5)$ & $15 \cdot 1$ to 22.9 \\
\hline
\end{tabular}

$\mathrm{CI}=$ confidence interval; $\mathrm{SVI}=$ severe visual impairment.

more difficult for rural children and they are probably under-represented. The children included in the study therefore represent those with bilateral SVI in whom this is the major disability and whose parents have awareness of an access to a school for the blind.

There is no national blindness registration system in India. A community based survey could provide data on prevalence and causes of visual impairment but a very large study would be required to estimate cause specific prevalence rates since the all cause prevalence of childhood blindness in India is estimated to be between 0.4 and $1 \cdot 0 / 1000$ children. ${ }^{8}$ Additionally, some of the population data required for sampling frames to plan such a study are incomplete for some regions of the country.

The proportion of SVI/blindness attributable to vitamin A deficiency in this study of 1318 children is $19 \%$. The clinical findings of corneal scarring, anterior corneal staphyloma or phthisis bulbi, in conjunction with corneal pathology in the other eye, were used to identify children with probable previous keratomalacia. In the absence of documented biochemical or histological evidence it is not possible to be certain that all these children had corneal pathology resulting from vitamin A deficiency. It is, however, the most probable cause in children in this setting. Overall, corneal diseases accounted for $26 \%$ (348) of children with SVI/blindness in this study. The other causes of corneal scarring were measles in 3\% (42); ophthalmia neonatorum in $1 \%$ (10); harmful traditional eye medicines in 1\% (11); and trauma and keratitis in the remaining $3 \%$ (40) (table 3).

Vitamin A deficiency is known to be associated with a high mortality. ${ }^{11}$ The children seen in this study of schools for the blind therefore represent those surviving the deficiency state and its associated mortality and probably represent a minority of all those children who develop keratomalacia. ${ }^{12}$

It is not possible to estimate the prevalence of vitamin A deficiency in the population from the study data. Lack of vitamin A is, however, the most common single cause of blindness in children in this study from India. Moreover, it is amenable to prevention unlike some of the other causes identified. In the majority of children with corneal scarring, diarrhoeal diseases or measles were associated with the onset of visual loss. Both diseases are public health problems in India and are potentially preventable.

Comparison with blind school studies in other countries shows the contribution of vitamin A deficiency to range from $50 \%-70 \%$ in East Africa (Ethiopia, Malawi, and Tanzania), ${ }^{13}$ to $25 \%-40 \%$ in West Africa (Ghana, Togo, and Benin), ${ }^{14}$ and $1 \%$ in Chile. ${ }^{14}$ This range probably reflects the differences in prevalence of vitamin A deficiency and its associated diseases. Measles, for example, is particularly important as a predisposing factor in African countries. ${ }^{13}$ Diarrhoeal diseases are known to be important in Asia. ${ }^{15}$ Such differences partly reflect the success of primary health care interventions such as measles immunisation, teaching of oral rehydration practices, and nutrition education programmes in operation in these countries.

The data for India suggest that marked regional variations between states exist. The observed differences between states may reflect true differences in the prevalence of vitamin A deficiency or in the diseases predisposing to it. The traditional view, based on studies in the 1970 s, is that vitamin A deficiency is seen most frequently in West Bengal and in the southern states - Tamil Nadu and Kerala. ${ }^{16}$ Since the 1970s various vitamin A prophylaxis schemes have been implemented at national and local level with varying degrees of success. This has resulted in a changing pattern in the presentation of clinical vitamin A deficiency, with keratomalacia being reported uncommonly in Kerala and in the northern states of Haryana, Punjab, and Himanchal Pradesh. ${ }^{16}$ The findings of this study appear to be consistent with this evolving pattern.

Between 1980 and 1989 the first phase of the Tamil Nadu integrated nutrition project was implemented with the aim of improving family nutrition and maternal and child health services. This succeeded in halving the prevalence of malnutrition in Tamil Nadu. ${ }^{2}$ It is of interest that while the overall proportion of blindness due to vitamin A deficiency in Tamil Nadu is $19 \%$ and therefore the same as the proportion for the country as a whole, variations are observed within the state with an urban-rural gradient from $8 \%$ to $30 \%$. It is possible that these observations reflect differences in awareness of and access to preventive health care between urban and rural populations. These observed variations highlight the importance of health policy evaluation not only at national and state levels but also at district and local community levels.

The standardised examination procedures and definitions of visual loss permit useful comparisons with studies performed elsewhere in the world and facilitate comparisons with

Table 3 Causes of corneal scarring in 348 children with SVI/blindness in nine states in India, $1993(n=1318)$

\begin{tabular}{lc}
\hline Cause & No (\% of total) with \\
SVI/blindness
\end{tabular}

SVI $=$ severe visual impairment. 
follow up studies using the same methodology that could be carried out in the future in India. The simple, quick, and inexpensive examination of children in blind schools can be used as an indicator to identify communities in which vitamin A deficiency is likely to be a significant problem. Subsequent detailed population based studies, targeted at high risk communities, using conjunctival impression cytology and serum retinol concentrations, can then be carried out to confirm subclinical and clinical vitamin A deficiency.

\section{Conclusion}

This study identifies vitamin A deficiency as the most common single cause of severe visual impairment and blindness in children in India. Regional variations are observed. Further surveillance of the causes of blindness in children entering schools for the blind may be a useful and simple indicator with which to monitor the impact of public health measures to combat vitamin A deficiency.

The authors thank the Tjissen Foundation and the CBM for their support for the study.

Anonymous. Vitamin A for measles [Editorial]. Lancet 1987; i: $1067-8$.

2 World Bank. Investing in health. World development report. Oxford: Oxford University Press, 1993.
3 Tielsch JM, Sommer A. The epidemiology of vitamin A deficiency and xerophthalmia. Annu Rev Nutr 1984; 4; 183-205.

4 Pratinidhi AK, Bapat VS, Shah U. Magnitude of vitamin A deficiency in primary school children of Sirur. Indian $\Im$ Pediatr 1984 ; 51: 423-6.

5 Sommer A, Katz J, Tarwotjo I. Increased risk of respiratory disease and diarrhoea in children with pre-existing mild vitamin A deficiency. Am $₹$ Clin Nutr 1984; 40: mild vitami

6 Mathur GP, Kushwaha KP. Vitamin A deficiency: a review. Indian F Pediatr 1987; 24: 573-81.

7 World Health Organisation. Adaptation of international classification of diseases, 1975 revision. Geneva: WHO, 1977.

8 Foster A, Gilbert CE. Epidemiology of childhood blindness Eye 1992; 6: 173-6.

9 International Council for the Education of the Visually Handicapped. Resource directory. International Council for the Education of the Visually Handicapped: Bensheim, Germany: April 1986.

10 Gilbert CE, Foster A, Thylefors B, Negrel DA. Childhood blindness: a new form for recording causes of visual loss in children. WHO Bulletin 1993; 71: 485-9.

11 Cohen N, Rahman H, Sprague J, Jalil MA, Leemhuis de Regt E, Mitra M. Prevalence and determinants of nutriRegt E, Mitra M. Prevalence and determinants of nutri-
tional blindness in Bangladeshi children. World Health tional blindness in Bang
Stat $O$ 1985; 38: $317-30$.

12 International Vitamin A Consultative Group (IVAGG). The symptoms and signs of vitamin $A$ deficiency and their relationship to applied nutrition. Washington, DC: The Nutrition Foundation, 1981

13 Foster A, Sommer A. Childhood blindness from corneal ulceration in Africa: causes, prevention and treatment. WHO Bulletin 1986; 64: 619-23.

14 Gilbert CE, Canovas R, Hogan M, Rao S, Foster A. Causes of childhood blindness: results from West Africa, South India and Chile. Eye 1993; 7: 184-8.

15 Khan MU, Haque E, Khan MR. Nutritional ocular diseases and their association with diarrhoea in Matlab, and their association with diarr.

16 Chandra DB, Kumar P. Blindness in rural Indian children. In: Holmes WJ, ed. Public health ophthalmology. The Hague: Dr W Junk BV, 1974 